\title{
STUDY ON ITERATIVE LEARNING CONTROL FOR RIEMANN-LIOUVILLE TYPE FRACTIONAL-ORDER SYSTEMS
}

\author{
Zi JiAN LUO AND JiN RONG WANG
}

\begin{abstract}
In this paper, we explore P-type and D-type learning laws for two classes of RiemannLiouville fractional-order controlled systems to track the varying reference accurately by adopting a few iterations in a finite time interval. Firstly, we establish open and closed-loop P-type convergence results in the sense of $(1-\alpha, \lambda)$-weighted norm $\|\cdot\|_{1-\alpha, \lambda}$ for Riemann-Liouville fractional-order system of order $0<\alpha<1$ with initial state learning. Secondly, we establish open and closed-loop D-type convergence results in the sense of $\lambda$-weighted norm $\|\cdot\|_{\lambda}$ for Riemann-Liouville fractional-order system of order $1<\alpha<2$ with initial state learning. Finally, two numerical examples are given to illustrate our theoretical results.
\end{abstract}

Mathematics subject classification (2010): 34A37, 93C15, 93C40.

Keywords and phrases: Fractional-order system, iterative learning control, P-type, D-type, weighted norm.

\section{Introduction}

Since Uchiyama [1] and Arimoto [2, 3] put forward the iterative learning control (ILC for short), ILC has been extended to tracking tasks with iteratively varying reference trajectories [4, 5, 6, 7]. There are some contribution on P-type and D-type iterative learning control for integer order ordinary differential equations $[8,9,10,11]$. The contribution [12] pointed out that how to deal with iteration-varying factors became a focus of ILC research.

Nowadays, fractional calculus plays an important role in various fields such as electricity, signal and image processing. And many fractional order controllers have so far been implemented to enhance the robustness and the performance of the control systems [13]. Furthermore, ILC of fractional-order systems has been attracted by many researchers since fractional-order systems can better describe the behavior of the object in control problems [14, 15, 16, 17].

Recently, existence theory of solutions to fractional differential equations involving Riemann-Liouville derivatives has been investigated in [18, 19, 20, 21]. Some researches about Caputo fractional controlled systems have obtained some interesting results $[22,23,24,25]$.

Hence, in this work, we mainly consider Riemann-Liouville fractional-order nonlinear system of $0<\alpha<1$ :

$$
\left\{\begin{array}{l}
\left(D_{0+}^{\alpha} x_{k}\right)(t)=\mu x_{k}(t)+f\left(t, x_{k}(t), u_{k}(t)\right), t \in J=[0, T], \mu<0, \\
\lim _{t \rightarrow 0+}\left(D_{0+}^{\alpha-1} x_{k}\right)(t)=x_{k}(0),
\end{array}\right.
$$


with a given nonlinear output $y_{k}$.

Meanwhile, we consider Riemann-Liouville fractional-order nonlinear system of $1<\alpha<2$ :

$$
\left\{\begin{array}{l}
\left(D_{0+}^{\alpha} x_{k}\right)(t)=\mu x_{k}(t)+f\left(t, x_{k}(t), u_{k}(t)\right), t \in J, \mu<0, \\
\lim _{t \rightarrow 0+}\left(D_{0+}^{\alpha-1} x_{k}\right)(t)=x_{k}(0), \\
\lim _{t \rightarrow 0+}\left(D_{0+}^{\alpha-2} x_{k}\right)(t)=\hat{x}_{k}(0),
\end{array}\right.
$$

with a given linear output $y_{k}$.

We explore iterative learning control for Riemann-Liouville fractional controlled systems, and establish the convergence analysis of two kinds of ILC for fractional differential systems via initial state learning and with initial state learing. We try to design some ILC law to generate the control input $u_{k}(\cdot)$ such that the fractional system output $y_{k}(\cdot)$ tracks the reference trajectories $y_{d}(\cdot)$ as accurately as possible as $k \rightarrow \infty$ uniformly on a finite time interval in the sense of $(1-\alpha, \lambda)$-weighted norm for P-type ILC and order $\alpha \in(0,1)$ and $\lambda$-weighted norm for D-type ILC and order $\alpha \in(1,2)$. The ILC scheme would not fix the initial value on the expected condition at the beginning of each iteration. So, we lead into initial state learning.

For system (1.1) $(0<\alpha<1)$, we consider the open-loop P-type ILC updating law with initial state learning:

$$
\left\{\begin{array}{l}
x_{k+1}(0)=x_{k}(0)+L e_{k}(0), \\
u_{k+1}(t)=u_{k}(t)+\gamma_{1} e_{k}(t),
\end{array}\right.
$$

where $y_{d}(t)$ be the iteratively varying reference trajectories and $e_{k}(t)=y_{d}(t)-y_{k}(t)$ denotes the tracking error, $L$ and $\gamma_{1}$ are unknown parameters to be determined. And closed-loop P-type ILC updating law with initial state learning:

$$
\left\{\begin{array}{l}
x_{k+1}(0)=x_{k}(0)+L e_{k}(0), \\
u_{k+1}(t)=u_{k}(t)+\gamma_{2} e_{k+1}(t),
\end{array}\right.
$$

where $L$ and $\gamma_{2}$ are unknown parameters to be determined.

For system (1.2) $(1<\alpha<2)$, we consider the open-loop D-type ILC updating law with initial state learning:

$$
\left\{\begin{array}{l}
x_{k+1}(0)=x_{k}(0)+L \dot{e}_{k}(0), \\
u_{k+1}(t)=u_{k}(t)+\gamma_{1} \dot{e}_{k}(t) .
\end{array}\right.
$$

And the closed-loop D-type ILC updating law with initial state learning:

$$
\left\{\begin{array}{l}
x_{k+1}(0)=x_{k}(0)+L \dot{e}_{k}(0), \\
u_{k+1}(t)=u_{k}(t)+\gamma_{2} \dot{e}_{k+1}(t) .
\end{array}\right.
$$

The results of the P-type ILC and D-type ILC for full nonlinear fractional differential systems are derived respectively. Examples are given in final section to demonstrate the application of our main results. 


\section{Preliminaries}

Denote $\mathscr{C}\left(J, \mathbb{R}^{n}\right)$ be the Banach space of vector-value continuous functions from $J \rightarrow \mathbb{R}^{n}$ endowed with the $\infty$-norm $\|x\|_{\infty}=\max _{t \in J}\|x(t)\|$ or $\lambda$-norm $\|x\|_{\lambda}=\max _{t \in J} e^{-\lambda t}$ $\|x(t)\|$. We introduce the set $\mathscr{C}_{1-\alpha}(J, \mathbb{R})=\left\{x \in \mathscr{C}((0, T], \mathbb{R}): \cdot{ }^{1-\alpha} x(\cdot) \in \mathscr{C}(J, \mathbb{R})\right\}$ (see [18, Page 4]) and define $(1-\alpha, \lambda)$-weighted norm $\|x\|_{1-\alpha, \lambda}=\max _{t \in J} t^{1-\alpha} e^{-\lambda t}\|x(t)\|$, $0<\alpha<1$. From [21], $\left(\mathscr{C}_{1-\alpha}(J, \mathbb{R}),\|\cdot\|_{1-\alpha, \lambda}\right)$ is also being a Banach space.

Definition 1. (see [18, Formula (2.1.1)]) The Riemann-Liouville fractional integrals $I_{a+}^{\alpha} f$ is defined by

$$
\left(I_{a+f}^{\alpha} f\right)(x):=\frac{1}{\Gamma(\alpha)} \int_{a}^{x} \frac{f(t)}{(x-t)^{1-\alpha}} d t, \quad(x>a ; \alpha>0),
$$

and the Riemann-Liouville fractional derivatives $D_{a+}^{\alpha} f$ is defined by

$$
\begin{aligned}
\left(D_{a+}^{\alpha} f\right)(x) & :=\left(\frac{d}{d x}\right)^{n}\left(I_{a+}^{n-\alpha} f\right)(x) \\
& =\frac{1}{\Gamma(n-\alpha)}\left(\frac{d}{d x}\right)^{n} \int_{a}^{x} \frac{f(t)}{(x-t)^{\alpha-n+1}} d t, \quad(x>a ; \alpha>0 ; n=[\alpha]+1),
\end{aligned}
$$

where $\Gamma(\cdot)$ is Gamma function and $f(\cdot)$ is an integrable function.

Definition 2. (see [26, Formula (4.1.1)]) The two-parameter function of the Mittag-Leffler type function is defined by

$$
E_{\alpha, \beta}(z)=\sum_{k=0}^{\infty} \frac{z^{k}}{\Gamma(\alpha k+\beta)}, \quad(\alpha>0, \beta \in \mathbb{R}, z \in \mathbb{R}) .
$$

Lemma 1. (see [27, Lemma 2] and [28, Lemma 2.7]) Let $\alpha \in(0,2]$ and $\beta>0$ be arbitrary. The functions $E_{\alpha}(\cdot), E_{\alpha, \alpha}(\cdot)$ and $E_{\alpha, \beta}(\cdot)$ are nonnegative, and for all $z<0$,

$$
E_{\alpha}(z):=E_{\alpha, 1}(z) \leqslant 1, \quad E_{\alpha, \alpha}(z) \leqslant \frac{1}{\Gamma(\alpha)}, \quad E_{\alpha, \beta}(z) \leqslant \frac{1}{\Gamma(\beta)} .
$$

Lemma 2. (see [26, Formula (4.3.1)]) The fractional-order differentiation or integral of the Mittag-Leffler function is

$$
\left(\frac{d}{d z}\right)^{m}\left[z^{\beta-1} E_{\alpha, \beta}\left(z^{\alpha}\right)\right]=z^{\beta-m-1} E_{\alpha, \beta-m}\left(z^{\alpha}\right), \quad(\alpha>0, m \geqslant 1) .
$$

The following two generalized Gronwall inequalities will be used in the sequel.

Lemma 3. (see [29, Theorem 1]) Suppose $\beta>0, m(t)$ is a nonnegative function locally integrable on $J$ and $n(t)$ is a nonnegative, nondecreasing continuous function 
defined on $J, n(t) \leqslant M$ (M is constant $)$, and suppose $y(t)$ is nonnegative and locally integrable on $J$ with

$$
y(t) \leqslant m(t)+n(t) \int_{0}^{t}(t-s)^{\beta-1} y(s) d s, t \in J .
$$

Then

$$
y(t) \leqslant m(t)+\int_{0}^{t}\left[\sum_{j=1}^{\infty} \frac{(n(t) \Gamma(\beta))^{j}}{\Gamma(j \beta)}(t-s)^{j \beta-1} m(s)\right] d s, t \in J .
$$

REMARK 1. Under the hypothesis of Lemma 3, let $m(t)$ be a nondecreasing function on $J$. Then we have

$$
y(t) \leqslant m(t) E_{\beta}\left(n(t) \Gamma(\beta) t^{\beta}\right) .
$$

Lemma 4. (see [30, Lemma 3.1] or [31]) Let $u(t)$ be a continuous function on $t \in J$ and let $v(t-\tau)$ be continuous and nonnegative on the triangle $0 \leqslant \tau \leqslant t$. Moreover, let $w(t)$ be a positive continuous and non-decreasing function on $t \in J$. If

$$
u(t) \leqslant w(t)+\int_{0}^{t} v(t-\tau) u(\tau) d \tau, t \in J
$$

then

$$
u(t) \leqslant w(t) e^{\int_{0}^{t} v(t-\tau) d \tau}, t \in J
$$

\section{Convergence analysis of P-type for $0<\alpha<1$}

We consider Riemann-Liouville fractional-order nonlinear system of $0<\alpha<1$ :

$$
\left\{\begin{array}{l}
\left(D_{0+}^{\alpha} x_{k}\right)(t)=\mu x_{k}(t)+f\left(t, x_{k}(t), u_{k}(t)\right), t \in J, \mu<0, \\
\lim _{t \rightarrow 0+}\left(D_{0+}^{\alpha-1} x_{k}\right)(t)=x_{k}(0), \\
y_{k}(t)=g\left(t, x_{k}(t), u_{k}(t)\right),
\end{array}\right.
$$

where $k$ denotes the $k$ th learning iteration. The nonlinear terms $f, g: J \times \mathbb{R} \times \mathbb{R} \rightarrow \mathbb{R}$. The variables $x_{k}(t), u_{k}(t), y_{k}(t) \in \mathbb{R}$ are denoted by state, input and output, respectively. In addition, $\lim _{t \rightarrow 0+}\left(D_{0+}^{\alpha-1} x_{k}\right)(t)=x_{k}(0)$ denotes initial condition and $D_{0+}^{\alpha-1}$ denotes Riemann-Liouville fractional derivative (see [18, Formula (4.1.2)]). Obviously, the solution of (3.1) equivalent to the solution of following integral equation (see [18, Formula (4.1.10)]):

$$
\begin{aligned}
x_{k}(t)= & t^{\alpha-1} E_{\alpha, \alpha}\left(\mu t^{\alpha}\right) x_{k}(0) \\
& +\int_{0}^{t}(t-s)^{\alpha-1} E_{\alpha, \alpha}\left(\mu(t-s)^{\alpha}\right) f\left(s, x_{k}(s), u_{k}(s)\right) d s, t \in J .
\end{aligned}
$$

We impose the following assumptions. 
$\left(A_{1}\right) f: J \times \mathbb{R} \times \mathbb{R} \rightarrow \mathbb{R}$ is jointly continuous. In addition, there exist nondecreasing and nonnegative continuous functions $L_{f}(\cdot)$ and $I_{f}(\cdot)$ such that

$$
\left\|f\left(t, x_{k}, u_{k}\right)-f\left(t, \hat{x}_{k}, \hat{u}_{k}\right)\right\| \leqslant L_{f}(t)\left\|x_{k}-\hat{x}_{k}\right\|+I_{f}(t)\left\|u_{k}-\hat{u}_{k}\right\|, \quad t \in J .
$$

$\left(A_{2}\right) g: J \times \mathbb{R} \times \mathbb{R} \rightarrow \mathbb{R}$ and for $\eta_{j}>0, j=1,2,3,4$, suppose

$$
\left\{\begin{array}{l}
0<\eta_{1} \leqslant g_{u}=\frac{\partial g\left(t, x_{k}, u_{k}\right)}{\partial u_{k}} \leqslant \eta_{2}, \\
0<\eta_{3} \leqslant g_{x}=\frac{\partial g\left(t, x_{k}, u_{k}\right)}{\partial x_{k}} \leqslant \eta_{4} .
\end{array}\right.
$$

$\left(A_{3}\right)$ Let $M_{L}=\max \left\{\sup _{t \in J} \frac{L_{f}(t)}{t^{1-\alpha}}, \sup _{t \in J} \frac{I_{f}(t)}{t^{1-\alpha}}\right\}$ and $M_{L}$ is a finite positive constant.

\subsection{Open-loop case}

LEMMA 5. (see [14, Lemma 3.2]) For fractional-order nonlinear system (3.1) and given reference $y_{d}(t)$, denote that $\Delta u_{k}(t)=u_{k+1}(t)-u_{k}(t), \Delta x_{k}(t)=x_{k+1}(t)-$ $x_{k}(t)$, if

$\max \left\{\left|1-\gamma_{1} \eta_{1}-L \eta_{3}\right|,\left|1-\gamma_{1} \eta_{1}-L \eta_{4}\right|,\left|1-\gamma_{1} \eta_{2}-L \eta_{3}\right|,\left|1-\gamma_{1} \eta_{2}-L \eta_{4}\right|\right\} \leqslant \sigma_{0}<1$,

where $\sigma_{0}$ is constant, then for all $t \in J$, and arbitrary initial input $u_{0}$, the open-loop P-type ILC updating law (1.3) guarantees that $\lim _{k \rightarrow \infty}\left\|e_{k}(0)\right\|_{\lambda}=0$.

THEOREM 1. Assumptions $\left(A_{1}\right)-\left(A_{3}\right)$ hold. For fractional-order nonlinear system (3.1) and given reference $y_{d}(t)$, if the assumptions in Lemma 5 are met, furthermore, suppose that

$$
\max \left\{\left|1-\gamma_{1} \eta_{1}\right|,\left|1-\gamma_{1} \eta_{2}\right|\right\} \leqslant \sigma_{1}<1,
$$

where $\sigma_{1}$ is constant, then for arbitrary initial input $u_{0}$, the open-loop P-type ILC updating law (1.3) guarantees that $y_{k}$ tends to $y_{d} \in \mathscr{C}(J, \mathbb{R})$ in the sense of the $(1-$ $\alpha, \lambda)$-weighted norm for a sufficiently large $\lambda>0$.

Proof. It follows from $e_{k}(t)=y_{d}(t)-y_{k}(t)$ and the mean value theorem [14, (10)],

$$
\begin{aligned}
e_{k+1}(t) & =y_{d}(t)-y_{k+1}(t)=y_{d}(t)-y_{k}(t)+y_{k}(t)-y_{k+1}(t) \\
& =e_{k}(t)+y_{k}(t)-y_{k+1}(t) \\
& =e_{k}(t)-g_{x}(\xi) \Delta x_{k}(t)-g_{u}(\xi) \Delta u_{k}(t),
\end{aligned}
$$

where $\xi(\cdot)=\left(\cdot, x_{k}(\cdot)+\theta x_{k}(\cdot), u_{k}(\cdot)+\theta u_{k}(\cdot)\right), \theta \in(0,1)$.

So we have

$$
\begin{aligned}
e_{k+1}(t) & =e_{k}(t)-g_{x}(\xi) \Delta x_{k}(t)-g_{u}(\xi)\left[u_{k+1}(t)-u_{k}(t)\right] \\
& =e_{k}(t)-g_{x}(\xi) \Delta x_{k}(t)-g_{u}(\xi) \gamma_{1} e_{k}(t) \\
& =\left[1-\gamma_{1} g_{u}(\xi)\right] e_{k}(t)-g_{x}(\xi) \Delta x_{k}(t) .
\end{aligned}
$$


Then, we can get

$$
\left\|e_{k+1}(t)\right\| \leqslant\left|1-\gamma_{1} g_{u}(\xi)\right|\left\|e_{k}(t)\right\|+\left|g_{x}(\xi)\right|\left\|\Delta x_{k}(t)\right\| .
$$

Next, we will deal with $\Delta x_{k}(t)$, according to (3.2), we have

$$
\begin{aligned}
\Delta x_{k}(t)= & t^{\alpha-1} E_{\alpha, \alpha}\left(\mu t^{\alpha}\right) \Delta x_{k}(0) \\
& +\int_{0}^{t}(t-s)^{\alpha-1} E_{\alpha, \alpha}\left(\mu(t-s)^{\alpha}\right)\left[f\left(s, x_{k+1}(s), u_{k+1}(s)\right)-f\left(s, x_{k}(s), u_{k}(s)\right)\right] d s .
\end{aligned}
$$

Using Lemma 1 and (3.3), we get

$$
\begin{aligned}
\left\|\Delta x_{k}(t)\right\| \leqslant & \frac{t^{\alpha-1}}{\Gamma(\alpha)}\left\|\Delta x_{k}(0)\right\|+\frac{1}{\Gamma(\alpha)} \int_{0}^{t}(t-s)^{\alpha-1} L_{f}(s)\left\|\Delta x_{k}(s)\right\| d s \\
& +\frac{1}{\Gamma(\alpha)} \int_{0}^{t}(t-s)^{\alpha-1} I_{f}(s)\left\|\Delta u_{k}(s)\right\| d s .
\end{aligned}
$$

Then,

$$
\begin{aligned}
t^{1-\alpha}\left\|\Delta x_{k}(t)\right\| \leqslant & \frac{1}{\Gamma(\alpha)}\left\|\Delta x_{k}(0)\right\|+\frac{t^{1-\alpha}}{\Gamma(\alpha)} \int_{0}^{t}(t-s)^{\alpha-1} L_{f}(s)\left\|\Delta x_{k}(s)\right\| d s \\
& +\frac{t^{1-\alpha}}{\Gamma(\alpha)} \int_{0}^{t}(t-s)^{\alpha-1} I_{f}(s)\left\|\Delta u_{k}(s)\right\| d s . \\
\leqslant & \frac{1}{\Gamma(\alpha)}\left\|\Delta x_{k}(0)\right\|+\frac{t^{1-\alpha}}{\Gamma(\alpha)} \int_{0}^{t}(t-s)^{\alpha-1} s^{1-\alpha} \frac{L_{f}(s)}{s^{1-\alpha}}\left\|\Delta x_{k}(s)\right\| d s \\
& +\frac{t^{1-\alpha}}{\Gamma(\alpha)} \int_{0}^{t}(t-s)^{\alpha-1} s^{1-\alpha} \frac{I_{f}(s)}{s^{1-\alpha}}\left\|\Delta u_{k}(s)\right\| d s .
\end{aligned}
$$

In the light of $\left(A_{3}\right)$, we obtain

$$
\begin{aligned}
t^{1-\alpha}\left\|\Delta x_{k}(t)\right\| \leqslant & \frac{1}{\Gamma(\alpha)}\left\|\Delta x_{k}(0)\right\|+\frac{t^{1-\alpha} M_{L}}{\Gamma(\alpha)} \int_{0}^{t}(t-s)^{\alpha-1} s^{1-\alpha}\left\|\Delta x_{k}(s)\right\| d s \\
& +\frac{t^{1-\alpha} M_{L}}{\Gamma(\alpha)} \int_{0}^{t}(t-s)^{\alpha-1} s^{1-\alpha}\left\|\Delta u_{k}(s)\right\| d s .
\end{aligned}
$$

Obviously,

$$
\begin{aligned}
\int_{0}^{t}(t-s)^{\alpha-1} s^{1-\alpha}\left\|\Delta u_{k}(s)\right\| d s & =\int_{0}^{t}(t-s)^{\alpha-1} s^{1-\alpha} e^{-\lambda s}\left\|\Delta u_{k}(s)\right\| e^{\lambda s} d s \\
& \leqslant\left\|\Delta u_{k}\right\|_{1-\alpha, \lambda} \int_{0}^{t}(t-s)^{\alpha-1} e^{\lambda s} d s .
\end{aligned}
$$

Note that the fact

$$
\begin{aligned}
\int_{0}^{t}(t-s)^{\alpha-1} e^{\lambda s} d s & =\int_{0}^{t} \omega^{\alpha-1} e^{\lambda(t-\omega)} d \omega=e^{\lambda t} \int_{0}^{t} \omega^{\alpha-1} e^{-\lambda \omega} d \omega \\
& =\frac{e^{\lambda t}}{\lambda^{\alpha}} \int_{0}^{\lambda t} v^{\alpha-1} e^{-v} d v \leqslant \frac{e^{\lambda t}}{\lambda^{\alpha}} \Gamma(\alpha),
\end{aligned}
$$


next, (3.7) become

$$
\int_{0}^{t}(t-s)^{\alpha-1} s^{1-\alpha}\left\|\Delta u_{k}(s)\right\| d s \leqslant\left\|\Delta u_{k}\right\|_{1-\alpha, \lambda} \frac{e^{\lambda t}}{\lambda \alpha} \Gamma(\alpha) .
$$

So, (3.6) become

$$
t^{1-\alpha}\left\|\Delta x_{k}(t)\right\| \leqslant m(t)+\frac{t^{1-\alpha} M_{L}}{\Gamma(\alpha)} \int_{0}^{t}(t-s)^{\alpha-1} s^{1-\alpha}\left\|\Delta x_{k}(s)\right\| d s,
$$

where $m(t)=\frac{1}{\Gamma(\alpha)}\left\|\Delta x_{k}(0)\right\|+\left\|\Delta u_{k}\right\|_{1-\alpha, \lambda} \frac{e^{\lambda t} t^{1-\alpha} M_{L}}{\lambda^{\alpha}}$ is nondecreasing.

By Remark 1, one obtains

$$
t^{1-\alpha}\left\|\Delta x_{k}(t)\right\| \leqslant\left[\frac{1}{\Gamma(\alpha)}\left\|\Delta x_{k}(0)\right\|+\left\|\Delta u_{k}\right\|_{1-\alpha, \lambda} \frac{e^{\lambda t} t^{1-\alpha} M_{L}}{\lambda^{\alpha}}\right] E_{\alpha}\left(M_{L} T\right) .
$$

And then

$$
t^{1-\alpha} e^{-\lambda t}\left\|\Delta x_{k}(t)\right\| \leqslant \frac{e^{-\lambda t}\left\|\Delta x_{k}(0)\right\|}{\Gamma(\alpha)} E_{\alpha}\left(M_{L} T\right)+\frac{t^{1-\alpha} M_{L}}{\lambda^{\alpha}}\left\|\Delta u_{k}\right\|_{1-\alpha, \lambda} E_{\alpha}\left(M_{L} T\right) .
$$

So we obtain

$$
\left\|\Delta x_{k}\right\|_{1-\alpha, \lambda} \leqslant \frac{\left\|\Delta x_{k}(0)\right\|_{\lambda}}{\Gamma(\alpha)} E_{\alpha}\left(M_{L} T\right)+\frac{T^{1-\alpha} M_{L}}{\lambda^{\alpha}} E_{\alpha}\left(M_{L} T\right)\left\|\Delta u_{k}\right\|_{1-\alpha, \lambda} .
$$

In case of $\left\|\Delta u_{k}\right\|_{1-\alpha, \lambda}=\gamma_{1}\left\|\Delta e_{k}\right\|_{1-\alpha, \lambda}$, and consider (3.5), we take $\|\cdot\|_{1-\alpha, \lambda}$, then

$$
\begin{aligned}
\left\|e_{k+1}\right\|_{1-\alpha, \lambda} \leqslant & \left|1-\gamma_{1} g_{u}(\xi)\right|\left\|e_{k}\right\|_{1-\alpha, \lambda}+\left|g_{x}(\xi)\right|\left\|\Delta x_{k}\right\|_{1-\alpha, \lambda} \\
\leqslant & \left|1-\gamma_{1} g_{u}(\xi)\right|\left\|e_{k}\right\|_{1-\alpha, \lambda} \\
& +\left|g_{x}(\xi)\right|\left[\frac{\left\|\Delta x_{k}(0)\right\|_{\lambda}}{\Gamma(\alpha)} E_{\alpha}\left(M_{L} T\right)+\frac{T^{1-\alpha} M_{L} \gamma_{1}}{\lambda^{\alpha}} E_{\alpha}\left(M_{L} T\right)\left\|\Delta e_{k}\right\|_{1-\alpha, \lambda}\right] \\
\leqslant & {\left[\left|1-\gamma_{1} g_{u}(\xi)\right|+\frac{T^{1-\alpha} M_{L} \gamma_{1}\left|g_{x}(\xi)\right|}{\lambda^{\alpha}} E_{\alpha}\left(M_{L} T\right)\right]\left\|e_{k}\right\|_{1-\alpha, \lambda} } \\
& +\frac{\left|g_{x}(\xi)\right| E_{\alpha}\left(M_{L} T\right)}{\Gamma(\alpha)}\left\|\Delta x_{k}(0)\right\|_{\lambda} \\
\leqslant & {\left[\left|1-\gamma_{1} g_{u}(\xi)\right|+\frac{T^{1-\alpha} M_{L} \gamma_{1}\left|g_{x}(\xi)\right|}{\lambda^{\alpha}} E_{\alpha}\left(M_{L} T\right)\right]\left\|e_{k}\right\|_{1-\alpha, \lambda} } \\
& +\frac{\left|g_{x}(\xi)\right| E_{\alpha}\left(M_{L} T\right)}{\Gamma(\alpha)}|L|\left\|e_{k}(0)\right\|_{\lambda} .
\end{aligned}
$$

By (3.4), there exists a sufficiently large $\lambda$ such that

$$
\left|1-\gamma_{1} g_{u}(\xi)\right|+\frac{T^{1-\alpha} M_{L} \gamma_{1}\left|g_{x}(\xi)\right|}{\lambda^{\alpha}} E_{\alpha}\left(M_{L} T\right) \leqslant \sigma_{1}+\frac{T^{1-\alpha} M_{L} \gamma_{1}\left|\eta_{4}\right| E_{\alpha}\left(M_{L} T\right)}{\lambda^{\alpha}}<1 .
$$

Therefore, from inequality (3.12) and Lemma 5, we have $\lim _{k \rightarrow \infty}\left\|e_{k}\right\|_{1-\alpha, \lambda}=0$. The proof is completed. 
REMARK 2. For system (3.1), if we assume that $\lim _{t \rightarrow 0+}\left(D_{0+}^{\alpha-1} x_{k}\right)(0)=x(0)$ and $L_{f}(t)=L_{f}, I_{f}(t)=I_{f}$ for $t \in J$, then the assumption $\left(A_{3}\right)$ can be removed. Moreover, we can derive that open-loop P-type ILC updating law (1.3) guarantees that $y_{k}$ tends to $y_{d}$ in the sense of $\lambda-$ norm for a sufficiently large $\lambda>0$. It means that

$$
\left\|e_{k+1}\right\|_{\lambda} \leqslant\left[\left|1-\gamma_{1} g_{u}(\xi)\right|+\frac{\left|\eta_{4}\right| I_{f}\left|\gamma_{1}\right| E_{\alpha}\left(L_{f} T^{\alpha}\right)}{\lambda^{\alpha}}\right]\left\|e_{k}\right\|_{\lambda}
$$

for a sufficiently large $\lambda$ such that

$$
\left|1-\gamma_{1} g_{u}(\xi)\right|+\frac{\left|\eta_{4}\right| I_{f}\left|\gamma_{1}\right| E_{\alpha}\left(L_{f} T^{\alpha}\right)}{\lambda^{\alpha}} \leqslant \sigma_{1}+\frac{\left|\eta_{4}\right| I_{f}\left|\gamma_{1}\right| E_{\alpha}\left(L_{f} T^{\alpha}\right)}{\lambda^{\alpha}}<1 .
$$

\subsection{Closed-loop case}

LEMMA 6. (see [14, Lemma 3.7]) For fractional-order nonlinear system (3.1) and given reference $y_{d}(t)$,

$$
\max \left\{\left|\frac{1-L \eta_{3}}{1+\gamma_{2} \eta_{1}}\right|,\left|\frac{1-L \eta_{3}}{1+\gamma_{2} \eta_{2}}\right|,\left|\frac{1-L \eta_{4}}{1+\gamma_{2} \eta_{1}}\right|,\left|\frac{1-L \eta_{4}}{1+\gamma_{2} \eta_{2}}\right|\right\} \leqslant \sigma_{2}<1,
$$

where $\sigma_{2}$ is constant, then for all $t \in J$, and arbitrary initial input $u_{0}$, the closed-loop P-type ILC updating law (1.4) guarantees that $\lim _{k \rightarrow \infty}\left\|e_{k}(0)\right\|_{\lambda}=0$.

THEOREM 2. Assumptions $\left(A_{1}\right)-\left(A_{3}\right)$ hold. For fractional-order nonlinear system (3.1) and given reference $y_{d}(t)$, if the assumptions in Lemma 6 are met, furthermore, suppose that

$$
\min \left\{\left|1+\gamma_{2} \eta_{1}\right|,\left|1+\gamma_{2} \eta_{2}\right|\right\} \geqslant \sigma_{3}>1
$$

where $\sigma_{3}$ is constant, then for arbitrary initial input $u_{0}$, the closed-loop P-type ILC updating law (1.4) guarantees that $y_{k}$ tends to $y_{d} \in \mathscr{C}(J, \mathbb{R})$ in the sense of the $(1-$ $\alpha, \lambda)$-weighted norm for a sufficiently large $\lambda>0$.

Proof. The proof is similar to Theorem 1, we present main different parts. Firstly,

$$
e_{k+1}(t)=e_{k}(t)-g_{x}(\xi) \Delta x_{k}(t)-g_{u}(\xi) \gamma_{2} e_{k+1}(t) .
$$

So, we derive that

$$
\left(1+g_{u}(\xi) \gamma_{2}\right) e_{k+1}(t)=e_{k}(t)-g_{x}(\xi) \Delta x_{k}(t)
$$

where $\xi(\cdot) \in\left(\cdot, x_{k}(\cdot)+\theta x_{k}(\cdot), u_{k}(\cdot)+\theta u_{k}(\cdot)\right), \theta \in(0,1)$.

And then, we obtain

$$
\left|1+g_{u}(\xi) \gamma_{2}\right|\left\|e_{k+1}(t)\right\| \leqslant\left\|e_{k}(t)\right\|+\left|g_{x}(\xi)\right|\left\|\Delta x_{k}(t)\right\|,
$$


which implies that

$$
\left|1+g_{u}(\xi) \gamma_{2}\right|\left\|e_{k+1}\right\|_{1-\alpha, \lambda} \leqslant\left\|e_{k}\right\|_{1-\alpha, \lambda}+\left|g_{x}(\xi)\right|\left\|\Delta x_{k}\right\|_{1-\alpha, \lambda} .
$$

It means that

$$
\left\|e_{k+1}\right\|_{1-\alpha, \lambda} \leqslant\left|\frac{1}{1+g_{u}(\xi) \gamma_{2}}\right|\left\|e_{k}\right\|_{1-\alpha, \lambda}+\left|\frac{g_{x}(\xi)}{1+g_{u}(\xi) \gamma_{2}}\right|\left\|\Delta x_{k}\right\|_{1-\alpha, \lambda} .
$$

As for $\left\|\Delta x_{k}\right\|_{1-\alpha, \lambda}$, we take formula (3.11) into (3.14), we obtain

$$
\begin{aligned}
\left\|e_{k+1}\right\|_{1-\alpha, \lambda} \leqslant & \left|\frac{1}{1+g_{u}(\xi) \gamma_{2}}\right|\left\|e_{k}\right\|_{1-\alpha, \lambda}+\left|\frac{g_{x}(\xi)}{1+g_{u}(\xi) \gamma_{2}}\right|\left[\frac{\left\|\Delta x_{k}(0)\right\|_{\lambda}}{\Gamma(\alpha)} E_{\alpha}\left(M_{L} T\right)\right. \\
& \left.+\frac{T^{1-\alpha} M_{L}\left|\gamma_{2}\right|}{\lambda^{\alpha}} E_{\alpha}\left(M_{L} T\right)\left\|e_{k+1}\right\|_{1-\alpha, \lambda}\right] \\
\leqslant & \left|\frac{1}{1+g_{u}(\xi) \gamma_{2}}\right|\left\|e_{k}\right\|_{1-\alpha, \lambda}+\left|\frac{g_{x}(\xi)}{1+g_{u}(\xi) \gamma_{2}}\right| \frac{\left\|\Delta x_{k}(0)\right\|_{\lambda}}{\Gamma(\alpha)} E_{\alpha}\left(M_{L} T\right) \\
& +\left|\frac{g_{x}(\xi)}{1+g_{u}(\xi) \gamma_{2}}\right| \frac{T^{1-\alpha} M_{L}\left|\gamma_{2}\right|}{\lambda^{\alpha}} E_{\alpha}\left(M_{L} T\right)\left\|e_{k+1}\right\|_{1-\alpha, \lambda} .
\end{aligned}
$$

Finally, we get

$$
\left\|e_{k+1}\right\|_{1-\alpha, \lambda} \leqslant \frac{\left\|e_{k}\right\|_{1-\alpha, \lambda}}{\left|1+g_{u}(\xi) \gamma_{2}\right|-\frac{g_{x}(\xi) c^{*}}{\lambda^{\alpha}}}+\frac{g_{x}(\xi) E_{\alpha}\left(M_{L} T\right) L\left\|e_{k}(0)\right\|_{\lambda}}{\left[\left|1+g_{u}(\xi) \gamma_{2}\right|-\frac{g_{x}(\xi) c^{*}}{\lambda^{\alpha}}\right] \Gamma(\alpha)},
$$

where $c^{*}=T^{1-\alpha} M_{L} E_{\alpha}\left(M_{L} T\right)\left|\gamma_{2}\right|$.

There exists a sufficiently large $\lambda$ such that $\varepsilon:=\frac{g_{x}(\xi) c^{*}}{\lambda^{\alpha}}>0$ is very small and $\sigma_{3}-\varepsilon>1$. Then by (3.13), one can obtain

$$
\zeta:=\frac{1}{\left|1+g_{u}(\xi) \gamma_{2}\right|-\frac{g_{x}(\xi) c^{*}}{\lambda^{\alpha}}} \leqslant \frac{1}{\sigma_{3}-\varepsilon}<1 .
$$

Therefore, from inequality (3.15) and Lemma 6, we have $\lim _{k \rightarrow \infty}\left\|e_{k}\right\|_{1-\alpha, \lambda}=0$. The proof is completed.

REMARK 3. For system (3.2), if we assume that $\lim _{t \rightarrow 0+}\left(D_{0+}^{\alpha-1} x_{k}\right)(0)=x(0)$ and $L_{f}(t)=L_{f}, I_{f}(t)=I_{f}$ for $t \in J$, then the assumption $\left(A_{3}\right)$ can be removed. Moreover, one can find that closed-loop P-type ILC updating law (1.4) guarantees that $y_{k}$ tends to $y_{d}$ in the sense of $\lambda-$ norm for a sufficiently large $\lambda$ satisfying

$$
\left|1+\gamma_{2} g_{u}(\xi)\right|-\frac{\eta_{4} I_{f}\left|\gamma_{1}\right| E_{\alpha}\left(L_{f} T^{\alpha}\right)}{\lambda^{\alpha}} \geqslant \sigma_{3}-\frac{\eta_{4} I_{f}\left|\gamma_{1}\right| E_{\alpha}\left(L_{f} T^{\alpha}\right)}{\lambda^{\alpha}}>1 .
$$




\section{Convergence analysis of D-type for $1<\alpha<2$}

We consider Riemann-Liouville fractional-order nonlinear system of order $1<$ $\alpha<2$ with impulses:

$$
\left\{\begin{array}{l}
\left(D_{0+}^{\alpha} x_{k}\right)(t)=\mu x_{k}(t)+f\left(t, x_{k}(t), u_{k}(t)\right), t \in J, \mu<0, \\
\lim _{t \rightarrow 0+}\left(D_{0+}^{\alpha-1} x_{k}\right)(t)=x_{k}(0), \\
\lim _{t \rightarrow 0+}\left(D_{0+}^{\alpha-2} x_{k}\right)(t)=\hat{x}_{k}(0), \\
y_{k}(t)=c x_{k}(t)+d \int_{0}^{t} u_{k}(s) d s, \quad c, d \in \mathbb{R} .
\end{array}\right.
$$

where $k$ denotes the $k$ th learning iteration, $T$ denotes pre-fixed iteration domain length. The nonlinear terms $f: J \times \mathbb{R} \times \mathbb{R} \rightarrow \mathbb{R}$. The variables $x_{k}(t), u_{k}(t), y_{k}(t) \in \mathbb{R}$ are denoted by state, input and output, respectively. In addition, $\lim _{t \rightarrow 0+}\left(D_{0+}^{\alpha-1} x_{k}\right)(t)=x_{k}(0)$ and $\lim _{t \rightarrow 0+}\left(D_{0+}^{\alpha-2} x_{k}\right)(t)=\hat{x}_{k}(0)$ denotes initial condition. $D_{0+}^{\alpha-1}$ and $D_{0+}^{\alpha-2}$ denotes Riemann-Liouville fractional derivative (see [18, Formula (4.1.2)]). With the initial condition, the solution of (4.1) equivalent to the solution of following integral equation (see [18, Formula (4.1.10)]):

$$
\begin{aligned}
x_{k}(t)= & x_{k}(0) t^{\alpha-1} E_{\alpha, \alpha}\left(\mu t^{\alpha}\right)+\hat{x}_{k}(0) t^{\alpha-2} E_{\alpha, \alpha-1}\left(\mu t^{\alpha}\right) \\
& +\int_{0}^{t}(t-s)^{\alpha-1} E_{\alpha, \alpha}\left(\mu(t-s)^{\alpha}\right) f\left(s, x_{k}(s), u_{k}(s)\right) d s .
\end{aligned}
$$

\subsection{Open-loop case}

THEOREM 3. Assumptions $\left(A_{1}\right)$ and $\left(A_{2}\right)$ with $L_{f}(t)=L_{f}, I_{f}(t)=I_{f}$, hold. Taking (1.5) into the fractional-order nonlinear system (4.1) and the initial condition at each iteration remains the desired, i.e., $e_{k}(0)=0, \dot{e}_{k}(0)=0$ and $\hat{x}_{k}(0)$ is constant, $k=0,1,2, \ldots$. If $\left|1-d \gamma_{1}\right|<1$, for arbitrary initial input $u_{0}(t)$, then the open-loop D-type ILC updating law (1.5) guarantees that $y_{k}$ tends to $y_{d} \in \mathscr{C}(J, \mathbb{R})$ in the sense of the $\lambda$ norm for a sufficiently large $\lambda>0$.

Proof. Note that

$$
\begin{aligned}
\dot{e}_{k+1}(t) & =\dot{y}_{d}(t)-\dot{y}_{k+1}(t)=\dot{e}_{k}(t)+c\left[\dot{y}_{k}(t)-\dot{y}_{k+1}(t)\right] \\
& =\dot{e}_{k}(t)+c\left[\dot{x}_{k}(t)-\dot{x}_{k+1}(t)\right]-d \Delta u_{k}(t) \\
& =\dot{e}_{k}(t)-d \gamma_{1} \dot{e}_{k}(t)+c\left[\dot{x}_{k}(t)-\dot{x}_{k+1}(t)\right] \\
& =\left(1-d \gamma_{1}\right) \dot{e}_{k}(t)+c\left[\dot{x}_{k}(t)-\dot{x}_{k+1}(t)\right] .
\end{aligned}
$$

Due to (4.2), Lemma 1, Lemma 2 and the conditions of Theorem 3, we obtain that

$$
\begin{aligned}
\dot{e}_{k+1}(t)= & \left(1-d \gamma_{1}\right) \dot{e}_{k}(t) \\
& +c \int_{0}^{t}(t-s)^{\alpha-2} E_{\alpha, \alpha-1}\left(\mu(t-s)^{\alpha}\right)\left[f\left(s, x_{k}(s), u_{k}(s)\right)-f\left(s, x_{k+1}(s), u_{k+1}(s)\right)\right] d s \\
\leqslant & \left(1-d \gamma_{1}\right) \dot{e}_{k}(t) \\
& +c \int_{0}^{t}(t-s)^{\alpha-2} \frac{1}{\Gamma(\alpha-1)}\left[f\left(s, x_{k}(s), u_{k}(s)\right)-f\left(s, x_{k+1}(s), u_{k+1}(s)\right)\right] d s .
\end{aligned}
$$


Note Lemma 1, and take norm on the above equality, then we can get

$$
\begin{aligned}
\left\|\dot{e}_{k+1}(t)\right\| \leqslant & \left|1-d \gamma_{1}\right|\left\|\dot{e}_{k}(t)\right\| \\
& +|c| \int_{0}^{t}(t-s)^{\alpha-2} \frac{1}{\Gamma(\alpha-1)}\left\|f\left(s, x_{k}(s), u_{k}(s)\right)-f\left(s, x_{k+1}(s), u_{k+1}(s)\right)\right\| d s \\
\leqslant & \left|1-d \gamma_{1}\right|\left\|\dot{e}_{k}(t)\right\|+\frac{|c|}{\Gamma(\alpha-1)} \int_{0}^{t}(t-s)^{\alpha-2} L_{f}\left\|\Delta x_{k}(s)\right\| d s \\
& +\frac{|c|}{\Gamma(\alpha-1)} \int_{0}^{t}(t-s)^{\alpha-2} I_{f}\left\|\Delta u_{k}(s)\right\| d s .
\end{aligned}
$$

Note that

$$
\begin{aligned}
\left\|\Delta x_{k}(s)\right\| \leqslant & \left\|s^{\alpha-1} E_{\alpha, \alpha}\left(\mu s^{\alpha}\right) \Delta x_{k}(0)\right\| \\
& +\int_{0}^{s}(s-\tau)^{\alpha-1} \frac{1}{\Gamma(\alpha-1)}\left\|f\left(\tau, x_{k}(\tau), u_{k}(\tau)\right)-f\left(\tau, x_{k+1}(\tau), u_{k+1}(\tau)\right)\right\| d \tau .
\end{aligned}
$$

Since $\Delta x_{k}(0)=L \dot{e}_{k}(0)=0$, we have

$$
\left\|\Delta x_{k}(s)\right\| \leqslant \frac{L_{f}}{\Gamma(\alpha-1)} \int_{0}^{s}(s-\tau)^{\alpha-1}\left\|\Delta x_{k}(\tau)\right\| d \tau+\frac{I_{f}}{\Gamma(\alpha-1)} \int_{0}^{s}(s-\tau)^{\alpha-1}\left\|\Delta u_{k}(\tau)\right\| d \tau .
$$

According to Lemma 4, we have

$$
\begin{aligned}
\left\|\Delta x_{k}(s)\right\| & \leqslant \frac{I_{f}}{\Gamma(\alpha-1)} \int_{0}^{s}(s-\tau)^{\alpha-1}\left\|\Delta u_{k}(\tau)\right\| d \tau \cdot e^{\int_{0}^{s} \frac{I_{f}}{\Gamma(\alpha-1)}(s-\tau)^{\alpha-1} d \tau} \\
& \leqslant \frac{I_{f}}{\Gamma(\alpha-1)} \int_{0}^{s}(s-\tau)^{\alpha-1}\left\|\Delta u_{k}(\tau)\right\| d \tau \cdot e^{\frac{I_{f} s^{\alpha}}{\alpha \Gamma(\alpha-1)}} \\
& \leqslant \frac{I_{f}}{\Gamma(\alpha-1)} \int_{0}^{s}(s-\tau)^{\alpha-1} e^{\lambda \tau} e^{-\lambda \tau}\left\|\Delta u_{k}(\tau)\right\| d \tau \cdot e^{\frac{I_{f} T^{\alpha}}{\alpha \Gamma(\alpha-1)}} \\
& \leqslant \frac{I_{f}}{\Gamma(\alpha-1)} \int_{0}^{s}(s-\tau)^{\alpha-1} e^{\lambda \tau} d \tau\left\|\Delta u_{k}\right\|_{\lambda} e^{\frac{I_{f} T^{\alpha}}{\alpha \Gamma(\alpha-1)}} \\
& \leqslant \frac{h I_{f}}{\alpha \Gamma(\alpha-1)} e^{\lambda s} s^{\alpha}\left\|\Delta u_{k}\right\|_{\lambda}, \quad h=e^{\frac{I_{f} T^{\alpha}}{\alpha \Gamma(\alpha-1)}} .
\end{aligned}
$$

Hence, we can obtain

$$
e^{-\lambda s}\left\|\Delta x_{k}(s)\right\| \leqslant \frac{h I_{f}}{\alpha \Gamma(\alpha-1)} s^{\alpha}\left\|\Delta u_{k}\right\|_{\lambda}
$$

which means that

$$
\left\|\Delta x_{k}\right\|_{\lambda} \leqslant \frac{h I_{f}}{\alpha \Gamma(\alpha-1)} T^{\alpha}\left\|\Delta u_{k}\right\|_{\lambda}
$$


And then, we have

$$
\begin{aligned}
L_{f} \int_{0}^{t}(t-s)^{\alpha-2}\left\|\Delta x_{k}(s)\right\| d s & =L_{f} \int_{0}^{t}(t-s)^{\alpha-2} e^{\lambda s} e^{-\lambda s}\left\|\Delta x_{k}(s)\right\| d s \\
& \leqslant L_{f} \int_{0}^{t}(t-s)^{\alpha-2} e^{\lambda s} d s\left\|\Delta x_{k}\right\|_{\lambda} \\
& \leqslant L_{f} \int_{0}^{t}(t-s)^{(\alpha-1)-1} e^{\lambda s} d s\left\|\Delta x_{k}\right\|_{\lambda} \\
& \leqslant L_{f}\left\|\Delta x_{k}\right\|_{\lambda} \frac{e^{\lambda t}}{\lambda^{\alpha-1}} \Gamma(\alpha-1) .
\end{aligned}
$$

Similarly, we have

$$
\begin{aligned}
I_{f} \int_{0}^{t}(t-s)^{\alpha-2}\left\|\Delta u_{k}(s)\right\| d s & \leqslant I_{f} \int_{0}^{t}(t-s)^{\alpha-2} e^{\lambda s} d s\left\|\Delta u_{k}\right\|_{\lambda} \\
& \leqslant I_{f} \int_{0}^{t}(t-s)^{(\alpha-1)-1} e^{\lambda s} d s\left\|\Delta u_{k}\right\|_{\lambda} \\
& \leqslant I_{f}\left\|\Delta u_{k}\right\|_{\lambda} \frac{e^{\lambda t}}{\lambda^{\alpha-1}} \Gamma(\alpha-1) .
\end{aligned}
$$

Taking (4.5) and (4.6) into (4.3), we get

$$
\left\|\dot{e}_{k+1}(t)\right\| \leqslant\left|1-d \gamma_{1}\right|\left\|\dot{e}_{k}(t)\right\|+\frac{e^{\lambda t} L_{f}|c|}{\lambda^{\alpha-1}}\left\|\Delta x_{k}\right\|_{\lambda}+\frac{e^{\lambda t} I_{f}|c|}{\lambda^{\alpha-1}}\left\|\Delta u_{k}\right\|_{\lambda} .
$$

So we get

$$
\left\|\dot{e}_{k+1}\right\|_{\lambda} \leqslant\left|1-d \gamma_{1}\right||| \dot{e}_{k}\left\|_{\lambda}+\frac{L_{f}|c|}{\lambda^{\alpha-1}}\right\| \Delta x_{k}\left\|_{\lambda}+\frac{I_{f}|c|}{\lambda^{\alpha-1}}\right\| \Delta u_{k} \|_{\lambda} .
$$

Taking (4.4) into (4.7), we get

$$
\begin{aligned}
\left\|\dot{e}_{k+1}\right\|_{\lambda} & \leqslant\left|1-d \gamma_{1}\right|\left\|\dot{e}_{k}\right\|_{\lambda}+\frac{h|c| L_{f} I_{f} T^{\alpha}}{\alpha \lambda^{\alpha-1} \Gamma(\alpha-1)}\left\|\Delta u_{k}\right\|_{\lambda}+\frac{I_{f}|c|}{\lambda^{\alpha-1}}\left\|\Delta u_{k}\right\|_{\lambda} \\
& \leqslant\left|1-d \gamma_{1}\right|\left\|\dot{e}_{k}\right\|_{\lambda}+\left[\frac{h|c| L_{f} I_{f} T^{\alpha}}{\alpha \lambda^{\alpha-1} \Gamma(\alpha-1)}+\frac{I_{f}|c|}{\lambda^{\alpha-1}}\right]\left\|\Delta u_{k}\right\|_{\lambda}, \quad h=e^{\frac{I_{f} T^{\alpha}}{\alpha \Gamma(\alpha-1)}} .
\end{aligned}
$$

Since we can choose $\lambda$ large enough such that $\left|1-d \gamma_{1}\right|<1$, we can get that $\lim _{k \rightarrow \infty}$ $\left\|\dot{e}_{k}\right\|_{\lambda}=0$. Since $e_{k}(0)=0, k=0,1,2, \ldots$, then we obtain $\lim _{k \rightarrow \infty}\left\|e_{k}\right\|_{\lambda}=0$. The proof is finished.

\subsection{Closed-loop case}

THEOREM 4. Assumptions $\left(A_{1}\right)$ and $\left(A_{2}\right)$ with $L_{f}(t)=L_{f}, I_{f}(t)=I_{f}$, hold. Taking (1.6) into the fractional-order nonlinear system (4.1) and the initial condition at each iteration remains the desired, i.e., $e_{k}(0)=0, \dot{e}_{k}(0)=0$ and $\hat{x}_{k}(0)$ is constant, $k=0,1,2, \ldots$. If $\left|\frac{1}{1+d \gamma_{2}}\right|<1$, for arbitrary initial input $u_{0}$, then the closed-loop $D$ type ILC updating law (1.5) guarantees that $y_{k}$ tends to $y_{d} \in \mathscr{C}(J, \mathbb{R})$ in the sense of the $\lambda$ norm for a sufficiently large $\lambda>0$. 
Proof. The main proof is similar to Theorem 3, we present the different steps. Note,

$$
\begin{aligned}
\dot{e}_{k+1}(t) & =\dot{e}_{k}(t)+c\left[\dot{x}_{k}(t)-\dot{x}_{k+1}(t)\right]-d \Delta u_{k}(t) \\
& =\dot{e}_{k}(t)-d \gamma_{2} \dot{e}_{k+1}(t)+c\left[\dot{x}_{k}(t)-\dot{x}_{k+1}(t)\right] \\
& =\frac{1}{1+d \gamma_{2}} \dot{e}_{k}(t)+\frac{c}{1+d \gamma_{2}}\left[\dot{x}_{k}(t)-\dot{x}_{k+1}(t)\right] .
\end{aligned}
$$

Then,

$$
\begin{aligned}
\left\|\dot{e}_{k+1}(t)\right\| \leqslant & \left|\frac{1}{1+d \gamma_{2}}\right|\left\|\dot{e}_{k}(t)\right\|+\left|\frac{c}{1+d \gamma_{2}}\right|\left\|\dot{x}_{k}(t)-\dot{x}_{k+1}(t)\right\| \\
\leqslant & \left|\frac{1}{1+d \gamma_{2}}\right|\left\|\dot{e}_{k}(t)\right\|+\left|\frac{c}{\left(1+d \gamma_{2}\right) \Gamma(\alpha-1)}\right| \int_{0}^{t}(t-s)^{\alpha-2} L_{f}\left\|\Delta x_{k}(s)\right\| d s \\
& +\left|\frac{c}{\left(1+d \gamma_{2}\right) \Gamma(\alpha-1)}\right| \int_{0}^{t}(t-s)^{\alpha-2} I_{f}\left\|\Delta u_{k}(s)\right\| d s .
\end{aligned}
$$

By adopting the same method of proving the Theorem 3 to deal with (4.8), we can get

$$
\begin{aligned}
\left\|\dot{e}_{k+1}(t)\right\| \leqslant & \left|\frac{1}{1+d \gamma_{2}}\right|\left\|\dot{e}_{k}(t)\right\|+\left|\frac{c}{\left(1+d \gamma_{2}\right) \Gamma(\alpha-1)}\right|\left\|\Delta x_{k}\right\|_{\lambda} \frac{e^{\lambda t}}{\lambda^{\alpha-1}} \Gamma(\alpha-1) \\
& +\left|\frac{c}{\left(1+d \gamma_{2}\right) \Gamma(\alpha-1)}\right|\left\|\Delta u_{k}\right\|_{\lambda} \frac{e^{\lambda t}}{\lambda^{\alpha-1}} \Gamma(\alpha-1) .
\end{aligned}
$$

And then, we have

$$
\begin{aligned}
\left\|\dot{e}_{k+1}\right\|_{\lambda} \leqslant & \left|\frac{1}{1+d \gamma_{2}}\right|\left\|\dot{e}_{k}\right\|_{\lambda}+\left|\frac{c}{1+d \gamma_{2}}\right|\left\|\Delta x_{k}\right\|_{\lambda} \frac{1}{\lambda^{\alpha-1}}+\left|\frac{c}{1+d \gamma_{2}}\right|\left\|\Delta u_{k}\right\|_{\lambda} \frac{1}{\lambda^{\alpha-1}} \\
\leqslant & \left|\frac{1}{1+d \gamma_{2}}\right|\left\|\dot{e}_{k}\right\|_{\lambda}+\left|\frac{c}{1+d \gamma_{2}}\right| \frac{h I_{f} T^{\alpha}}{\alpha \Gamma(\alpha-1)}\left\|\Delta u_{k}\right\|_{\lambda} \frac{1}{\lambda{ }^{\alpha-1}} \\
& +\left|\frac{c}{1+d \gamma_{2}}\right|\left\|\Delta u_{k}\right\|_{\lambda} \frac{1}{\lambda^{\alpha-1}} \\
\leqslant & \left|\frac{1}{1+d \gamma_{2}}\right|\left\|\dot{e}_{k}\right\|_{\lambda}+\left[\left|\frac{c}{1+d \gamma_{2}}\right| \frac{h I_{f} T^{\alpha}}{\alpha \Gamma(\alpha-1) \lambda^{\alpha-1}}+\left|\frac{c}{1+d \gamma_{2}}\right| \frac{1}{\lambda^{\alpha-1}}\right] \gamma_{2}\left\|\dot{e}_{k+1}\right\|_{\lambda} .
\end{aligned}
$$

So we have

$$
\left\|\dot{e}_{k+1}\right\|_{\lambda} \leqslant\left\{1-\left[\left|\frac{c}{1+d \gamma_{2}}\right| \frac{h I_{f} T^{\alpha} \gamma_{2}}{\alpha \Gamma(\alpha-1) \lambda^{\alpha-1}}+\left|\frac{c}{1+d \gamma_{2}}\right| \frac{\gamma_{2}}{\lambda^{\alpha-1}}\right]\right\}^{-1}\left|\frac{1}{1+d \gamma_{2}}\right|\left\|\dot{e}_{k}\right\|_{\lambda} .
$$

Due to the fact that exist a sufficiently large $\lambda$, and $\left|\frac{1}{1+d \gamma_{2}}\right|<1$, such that

$$
\left\{1-\left[\left|\frac{c}{1+d \gamma_{2}}\right| \frac{h I_{f} T^{\alpha} \gamma_{2}}{\alpha \Gamma(\alpha-1) \lambda^{\alpha-1}}+\left|\frac{c}{1+d \gamma_{2}}\right| \frac{\gamma_{2}}{\lambda^{\alpha-1}}\right]\right\}^{-1}\left|\frac{1}{1+d \gamma_{2}}\right|<1
$$

we can get that $\lim _{k \rightarrow \infty}\left\|\dot{e}_{k}\right\|_{\lambda}=0$. Then we obtain $\lim _{k \rightarrow \infty}\left\|e_{k}\right\|_{\lambda}=0$. The proof is finished. 


\section{Simulation examples}

In this section, two numerical examples are presented to demonstrate the validity of the designed method. In order to describe the stability of the system which is associated with the increase of iterations, we denote the total energy in $k$ th iteration as $\mathscr{E}_{k}=\left\|u_{k}\right\|_{\infty}$.

EXAMPLE 5.1. Consider

$$
\left\{\begin{array}{l}
\left(D_{0+}^{0.5} x_{k}\right)(t)=-x_{k}(t)+0.5(t+1) u_{k}(t), \\
\lim _{t \rightarrow 0+}\left(D_{0+}^{-0.5} x_{k}\right)(t)=0 \\
y_{k}(t)=x_{k}(t)+1.2 u_{k}(t)
\end{array}\right.
$$

and P-type ILC

$$
\left\{\begin{array}{l}
x_{k+1}(0)=x_{k}(0)+0.5 e_{k}(0), \\
u_{k+1}(t)=u_{k}(t)+e_{k}(t) .
\end{array}\right.
$$

Set $u_{k}(0)=0, \gamma_{1}=1$ and $\eta_{1}=\eta_{2}=d=1.2$. Obviously, $\left|1-\gamma_{1} \eta_{1}\right|=0.2<$ 1. All the conditions of Theorem 1 are satisfied. The original reference trajectory is $r(t)=3 t^{1.5}\left(t^{2}-t^{0.5}+1\right)$.

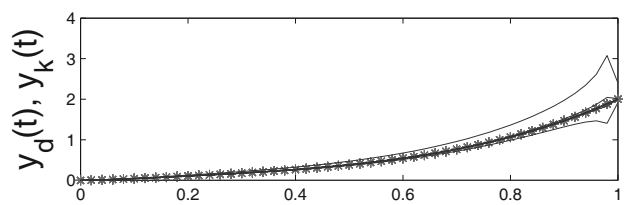

$t(s)$

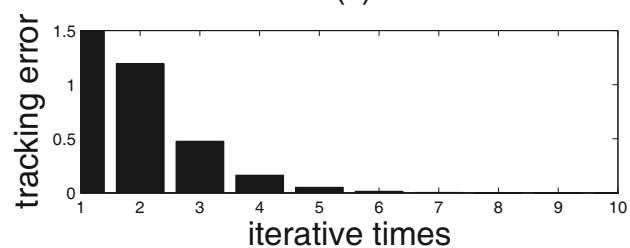

Figure 1: The system output and the tracking error.

The upper figure of Figure 1 shows the equation (5.1) output $y_{k}$ of the 10th iterations and the reference trajectory $y_{d}$. The lower figure of Figure 1 shows the $\infty$-norm of the tracking error in each iteration. 
EXAMPLE 5.2. Consider

$$
\left\{\begin{array}{l}
\left(D_{0+}^{1.5} x_{k}\right)(t)=-x_{k}(t)+\sin (t) u_{k}(t), \\
\lim _{t \rightarrow 0+}\left(D_{0+}^{0.5} x_{k}\right)(t)=0, \\
\lim _{t \rightarrow 0+}\left(D_{0+}^{-0.5} x_{k}\right)(t)=0 \\
y_{k}(t)=x_{k}(t)+1.2 \int_{0}^{t} u_{k}(s) d s,
\end{array}\right.
$$

and D-type ILC

$$
\left\{\begin{array}{l}
x_{k+1}(0)=x_{k}(0)+0.5 \dot{e}_{k}(0), \\
u_{k+1}(t)=u_{k+1}(t)+0.6 \dot{e}_{k}(t) .
\end{array}\right.
$$

Set $u_{k}(0)=0, \hat{x}_{k}(0)=0, \gamma_{1}=0.6$ and $d=1.2$. Obviously, $\left|1-d \gamma_{1}\right|=0.28<$ 1. All the conditions of Theorem 3 are satisfied. The original reference trajectory is $r(t)=3 t^{2}(t-1)$.
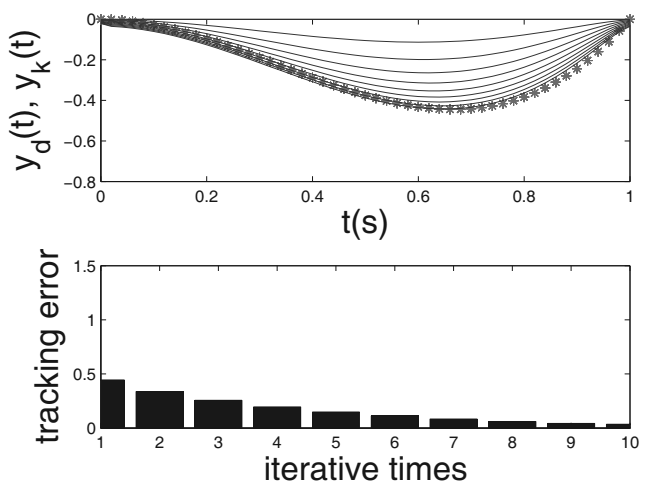

Figure 2: The system output and the tracking error.

The upper figure of Figure 2 shows the equation (5.2) output $y_{k}$ of the 10th iterations and the reference trajectory $y_{d}$. The lower figure of Figure 2 shows the $\infty$-norm of the tracking error in each iteration.

\section{Conclusion}

In this paper, iterative learning control for two class of Riemann-Liouville type fractional-order differential systems (one is $0<\alpha<1$, the other is $1<\alpha<2$ ) are firstly introduced to tracking the target with initial state offset. Secondly, the techniques of generalized Grownwall inequality and properties of Mittag-Leffler type functions are utilized to make estimation of the error. Finally, sufficient conditions for iterative learning law convergence are given in the sense of $(1-\alpha, \lambda)$-weighted norm and $\lambda$ weighted norm. 


\section{REFERENCES}

[1] M. UchiYama, Formulation of hirh-speed motion pattern of a mechanical arm by trial, Trans. Soc. Instrum. Contr. Eng. 6, 14 (1978), 706-712.

[2] S. ARImoto And S. KaWAmURa, Bettering operation of robots by learning, J. Robotic syst. 2, 1 (1984), 123-140.

[3] S. ARimoto, Mathematical Theory of Learning with Applications to Robot Control, Adaptive and Learning Systems, Springer US, 1986.

[4] S. S. SAAB, W. G. VogT AND M. H. MiCKLE, Learning control algorithm for tracking slowly varying trajectories, IEEE Trans. Syst. Man and Cybernetics, Part B: Cybernetics, 4, 27 (1997), 657669.

[5] Y. Q. CHEN AND K. L. MoORE, Harnessing the nonrepetitiveness in iterative learning control, Proceedings of 41st IEEE Conf. Decision Control, Las Vegas, NV, U.S.A., 2002, 3350-3355.

[6] J. X. XU AND J. XU, On iterative learning for different tracking tasks in the presence of time-varying uncertainties, IEEE Trans. Syst. Man and Cybernetics, Part B: Cybernetics, 1, 34 (2004), 589-597.

[7] H. S. Ahn, K. L. Moore And Y. Q. Chen, Iterative Learning Control: Robustness and Monotonic Convergence for Interval Systems, Communications and Control Engineering Series, Springer, Berlin, 2007.

[8] Z. BIEn And J. X. XU, Iterative Learning Control: Analysis, Design, Integration and Applications, Springer, New York, 1998.

[9] Y. Q. Chen AND C. Wen, Iterative Learning Control: Convergence, Robustness and Applications, Springer-Verlag, London, 1999.

[10] J. X. XU, A survey on iterative learning control for nonlinear systems, Int. J. Control 7, 84 (2011), $1275-1294$.

[11] J. V. DE WIJDEVEN, T. DONKERS AND O. BOSGRA, Iterative learning control for uncertain systems: Robust monotonic convergence analysis, Automatica 10, 45 (2009), 2383-2391.

[12] K. L. Moore, Y. Q. Chen And H. S. Ahn, Iterative learning control: Atutorial and big picture view, in Proc. 45th IEEE Conf. Decision Control, San Diego, CA, 2006, 2352-2357.

[13] Y. LUO AND Y. Q. CHEN, Fractional order controller for a class of fractional order systems, Automatica 10, 45 (2009), 2446-2450.

[14] Y. H. LAN, Iterative learning control with initial state learning for fractional order nonlinear systems, Comp. Math. Appl. 10, 64 (2012), 3210-3216.

[15] Y. H. LAN AND Y. ZHOU, $D^{\alpha}$-type iterative learning control for fractional order linear time-delay systems, Asian J. Control. 3, 15 (2013), 669-677.

[16] Y. LI, Y. Q. CHEN, H. S. AHN AND G. TIAN, A survey on fractional-order iterative learning control, J. Optim. Theory Appl. 1, 156 (2013), 127-140.

[17] Y. Li AND W. JiAng, Fractional order nonlinear systems with delay in iterative learning control, Appl. Math. Comput. C, 257 (2015), 546-552.

[18] A. A. Kilbas, H. M. SRivastava And J. J. Trujillo, Theory and Applications of Fractional Differential Equations, Elsevier, Amsterdam, 2006.

[19] C. BAI, Impulsive periodic boundary value problems for fractional differential equation involving Riemann-Liouville sequential fractional derivative, J. Math. Anal. Appl. 2, 384 (2011), 211-231.

[20] K. Li, J. Peng And J. JiA, Cauchy problems for fractional differential equations with RiemannLiouville fractional derivatives, J. Funct. Anal. 2, 263 (2012), 476-510.

[21] T. Jankowshi, Fractional equations of Volterra type involving a Riemann-Liouville derivative, Appl. Math. Lett. 26, 26 (2013), 344-350.

[22] Y. LI, Y. Q. CHEN AND H. S. AHN, A survey on fraction-order iterative learning control, J. Optim. Theory Appl. 1, 156 (2013), 127-140.

[23] Y. LI, Y. Q. Chen AND H. S. AHN, Fractional-order iterative learning control for fractional-order linear systems, Asian J. Control 1, 13 (2011), 1-10.

[24] C. F. Lorenzo And T. T. HARTLEy, Initialization of fractional-order operators and fractional differential equations, J. Comput. Nonlinear Dynam. 2, 3 (2008), 020201.1-021404.7.

[25] J. C. Trigeassou, N. MaAmri, J. Sabatier And A. Oustaloup, Transients of fractional-order integrator and derivatives, Signal, Image and Video Processing 3, 6 (2012), 1-14.

[26] R. Gorenflo, A. A. Kilbas, F. Mainardi and S. V. Rogosin, Mittag-Leffler Functions, Related Topics and Applications, Spring, Berlin, 2014. 
[27] J. WAng, M. FeČKAN AND Y. Zhou, Presentation of solutions of impulsive fractional Langevin equations and existence results, Eur. Phys. J. Special Topics 8, 222 (2013), 1857-1874.

[28] H. WANG, Existence of solutions for fractional anti-periodic BVP, Results. Math. 1-2, 68 (2015), 227-245.

[29] H. Ye, J. Gao And Y. Ding, A generalized Gronwall inequality and its application to a fractional differential equation, J. Math. Anal. Appl. 2, 328 (2007), 1075-1081.

[30] Y. Li AND Y. Q. CHEN, Iterative learning control of a class of fractional order nonlinear systems, IEEE International Symposium on Intelligent Control, Yokohama, Japan, 2010, 779-782.

[31] D. D. BAinov AND P. S. Simeonov, Integral inequalities and applications, Kluwer Academic Publishers, Dordrecht, 1992. 